\title{
Image Enhancement for the 3-D Reconstruction in the Uncontrolled Environment using Shape from Silhouette
}

\author{
Muhammad Sohaib \\ University of Engineering and \\ Technology, Peshawar, \\ Pakistan
}

\author{
Nasir Ahmed \\ University of Engineering and \\ Technology, Peshawar, \\ Pakistan
}

\author{
Nasru Minallah \\ University of Engineering and \\ Technology, Peshawar, \\ Pakistan
}

\begin{abstract}
Among the multiple models for 3-D shape reconstruction, Shape from silhouette (SFS) is one of the fast and simple 3D shape rendering techniques as compare to other approaches. In SFS model multiple images captured from different viewpoints in a controlled environment are used as input data at the front end to extract silhouette and are free of noises. Silhouette extraction from such well-defined input data is easy and accurate, having no loss of information while extracting the silhouette. On the other hand images from uncontrolled environment involve many degradation factors. Common and frequently degradation factors are motion blur and noise addition which effects acquired image quality, illumination and resolution seriously. The proposed work is an effort to extract useful information from such environmentally variant images. The successful reconstruction of the image is main emphasis.
\end{abstract}

\section{General Terms}

3D Modeling, Image Enhancement, Degradation Reduction

\section{Keywords}

3D reconstruction, silhouette, SFS pipelining, additive noise, blurriness

\section{INTRODUCTION}

Shape from Silhouette is one of the available 3D shape reconstruction techniques. In this, images from multiple viewpoints are captured and silhouette information is extracted from each picture. Later on using silhouette information and camera matrix generated from camera locations along with the subsequent steps of SFS pipeline well approximate resembled 3D model to the original object shape is reconstructed[1].Silhouette is the feature less expression of an object shape. Silhouette extraction from the input set of images is the basic and initial step in SFS 3D shape reconstruction pipeline. Accurate extraction of silhouette is needed for good quality 3D shape reconstruction. Images used for this purpose are of high details and are captured in controlled environment having uniform and known background. So they are of uniform illumination, high resolution, and are free of other kind of degradations. Acquiring object shape from such well-defined image is an easier. On the other hand photographs taken in an open environment or images acquire after traversing through some transmission media are exposed to different kind of noises and degradations. In this paper contribution to the front end of the Shape from Silhouette is made to propose an invariant system for environmentally degraded images.
SFS pipeline used for our work is obtained from [10]. The pipeline works on the basis space carving concept. First silhouettes are extracted from Input image. Silhouette extraction step is followed by volumetric reconstruction of shape thorough voxels. After the shape reconstruction non object voxels are removed from convex region by space carving also known as refinement step. Finally coloring of refined 3-D shape is done by surface coloring. Images and camera calibration matrix are acquired from University of Oxford Robotics Research Group [9].

Image degradation is defined as the process due to which unwanted information is added in image. Degradation may occur during image accusation or unwanted information is added during the process of transmission in remote imaging scenarios. A simple degradation process is defined as under.

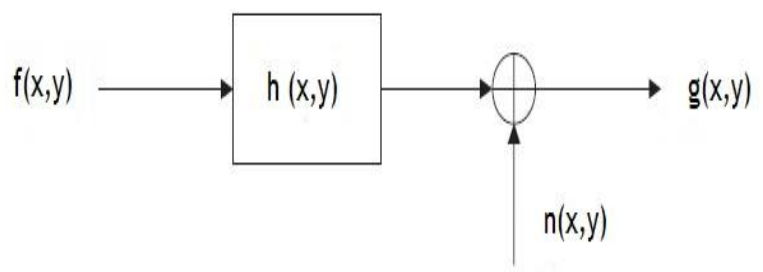

Fig. 1: A simple, Image degradation Model

In Figure 1, $g(x, y)$ is the resulted image after input image $f(x$, $\mathrm{y})$ passes through degradation function $\mathrm{h}(\mathrm{x}, \mathrm{y})$ and addition of noise $\mathrm{n}(\mathrm{x}, \mathrm{y})$. In our case degradation function is blurriness and noise function is additive and spatial noises.

Digital Imaging enabled human beings to develop photographs to Mirror scene or some kind of information of an object frequently, anywhere they want. Real Time picture is the ideal representation of the observed scene.

Although digital photography is quite sophisticated and well matured that can produce excellent level of results, but the observation process is still not ideal or error free. Many factors easily introduce noise or any other kind of degradation in a captured image. Blurriness is one of such unwanted phenomenon, which introduces unwanted information in the image. Most common factors that introduce blurriness in the images are camera shake and defocused image. Silhouette extraction from such degraded is difficult and leads to ambiguous information. Consequently, the developed 3D model at the end of the SFS process deviates from its original shape.

Noise is an additive process. It causes color or brightness variation in the image. Impulsive noise added during 
photography due to unwanted electronic signal fluctuation is also known as spikes or Fat-tail distributed noise. Such kind of fluctuations is due to faulty apparatus or sensor of digital camera. Noise density can vary in image plane, from less to seriously damaged image. Image infected with high density noise are unsuitable as input to the SFS process, because silhouette extracted at the very first stage of the process is imperfect which leads to imperfect 3D model reconstruction.

\section{RELATED WORK}

There had been quite a handful of research done on 3D and still it is the emphasis of many researches. Different researches were looked forward to for the understanding and limitations of the field.

In [1-4], there is a brief insight to the study relating to $3 \mathrm{D}$ shape reconstruction through SFS method. SFS method uses a basic principal of Visual Hull. Visual Hull is the concept of 3D shape reconstruction by SFS method. Basic principal of 3D reconstruction through Visual Hull is to use silhouettes of an object observed through different viewpoints. Projection of the silhouette obtained forms a visual cone. The intersection of these visual cones of multiple silhouettes from different viewpoints forms a visual hull, which describes a real object 3D shape.

The shape reconstruction and volume estimation through SFS framework was explained in [1]. The proposed method was able to develop colored 3-D shapes. A method was proposed for 3-D shape reconstruction for highly reflective objects. It compared the proposed SFS method efficiency with that of 3D laser scanners. Another work [2] identified the problems concerned with the 3D shape estimation of non-convex objects. It identified the problem of finding which part of a non-convex object is relevant for $3 \mathrm{D}$ shape estimation and introduces concept of geometric Visual Hull to identify the non-convex regions of an observed shape. In [3] theory of SFS across time is presented. The proposed work deals with 3D shape reconstruction of objects with unknown motion by introducing the concept of Bounding Edge to represent the Visual Hull and alignment of Visual Hulls. The work carried out in [4] describes the concept of space carving to estimate 3D model in more realistic with usage of interaction between occlusion, shading and shape dependency on viewpoint.

In [5] survey on noise removal from images using different types of median filters is the subject. In this work different derivatives of Simple Adaptive Median filter have been applied to digital images to reduce noise level. After the application of different variants of SAM filter, the conclusion was drawn that among these filters Circular SAM filter was complex to implement with overhead of increased execution time. In [6] a modified version of median filter which can be applied on colored images preserving edge pixel information of an object was proposed. Different types of blind deconvolution techniques for motion removal and blurred image restoration are described in detail in [7-8]. In [11] angle of blur is estimation by, Cepstrum, Gaussian and Radon Transform. PSF estimation through robust multichannel blind de-convolution is carried out in [12]. In [13], blur kernel estimation through radon transform is defined. In this work blur kernel is estimated by two methods. In first method blur kernel is estimated by directly inverting the transform. From this computational efficiency is achieved. In the second method radon transform along with MAP framework is applied, to compute the blur kernel simultaneously.

\section{METHODOLOGY}

In the proposed system spatially degraded images are given as input to the SFS pipeline. In order to detect object shape from degraded images an enhancement mechanism as preprocessing step is introduced to the pre-developed SFS pipeline [10].

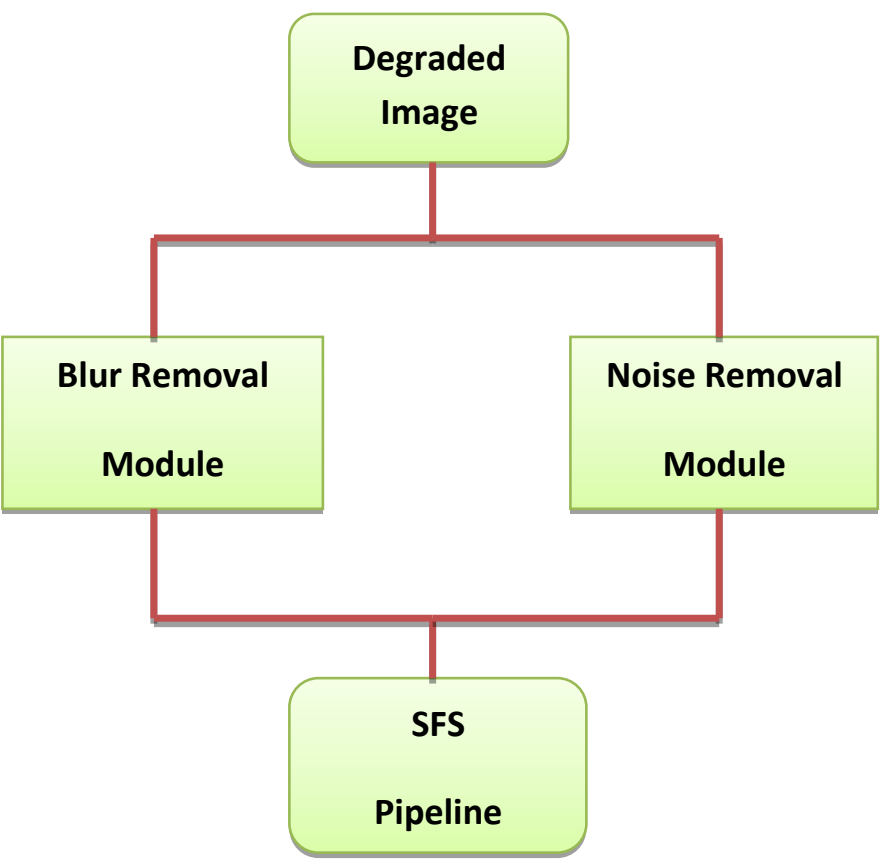

Fig. 2: Proposed System design

Figure 2 describes the proposed system design for the given problem. In proposed system design some modules are added as preprocessing steps which are as fallow. The modules added are Blurriness removal module and Noise removal module. These are explained as under.

\subsection{Blurriness Removal Module}

Blurriness removal from seriously defected images is difficult task. Here linear motion and out of focus blur are taken under consideration. Linear motion is caused by relative camera motion to the observed scene or motion in the object observed and is measured through PSF [8]. Another reason for blur image taken from digital camera is due to out of focus photography, which depends on many parameters itself i.e. focal length, distance between object observed and camera. There are many algorithms adopted for this purpose. Blurred image $\mathrm{g}(\mathrm{x}, \mathrm{y})$ is explained as

$$
\mathrm{g}(\mathrm{x}, \mathrm{y})=\mathrm{k} \otimes \mathrm{x}
$$

In above expression $\mathrm{k}$ is the blur kernel and $\mathrm{x}$ is considered as original image. For experiments we have adopted joint identification method [7] of blind de-convolution for parameter estimation and de-blurring given set of degraded images as input. In which blur and image are identified simultaneously. Here we have pre estimated parameters. Using this information de-convolution using cepstral [8] process is carried out. Cepstral method works on the basis of Fourier domain analysis [11-13].Cepstrum of a Blurred image $\mathrm{g}(\mathrm{x}, \mathrm{y})$ is calculated as fallow: 


$$
C_{x y}\left(g\left(x_{v} y\right)=F^{-1}\left(\log \left|F\left(g\left(x_{v} y\right)\right)\right|\right)\right.
$$

The above equation show that the cepstrum of an input image $\mathrm{g}(\mathrm{x}, \mathrm{y})$ can be estimated by taking Fourier transform of logarithmic estimated spectrum of an input data.

\subsection{Noise Removal Module}

In this module image artificially degraded image from impulsive noise is taken into consideration. In literature there are many algorithms developed to eliminate impulsive noise. Here we have used two dimensional median filtering to eliminate unwanted noise. 2-D Median filter is $[\mathrm{m} \times \mathrm{n}]$ nonlinear filter [5-6] and is more effective then convolution and preserve edge information more effectively. Instead of applying 2-D globally, a modified version is used, in which first three basic channels of colored picture are extracted and then to every channel 2-D median filter is applied. Results generated by the application of the filter to each and every channel separately and then concatenate three noise free channels are far better than its global application.

\subsection{SFS Pipeline}

In this section discussion about SFS Pipeline is carried out. The Pipeline consists of some sequential steps. Some of which are grouped as Front End and the latter are as Back End on the basis of their functionality. Figure 3 shows the front End of SFS Pipeline. Front End consists of three steps, camera calibration, image acquisition, silhouette extraction.

In camera calibration $3 \times 4$ camera matrix is calculated, which represents the true camera parameters for the reconstruction of 3D model. Camera calibration step is followed by image acquisition step. In this step object images are acquired from multiple view points by image acquisition system of high resolution. Then silhouette of the object is extracted from each of the captured images.

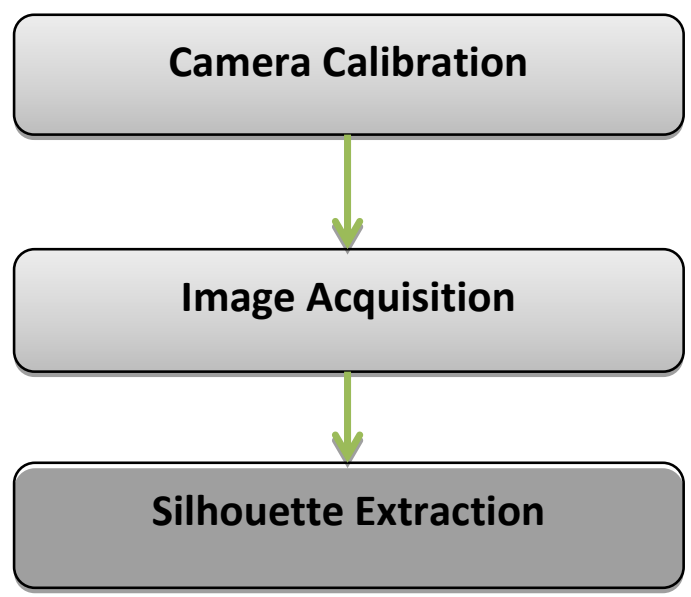

Fig. 3: Front End of SFS Pipeline

Figure 4 describes Back End of SFS Pipeline. Back End consists of shape reconstruction, removal of non-object voxels, refinement and shape evolution steps.

In shape reconstruction step rough 3D model estimation of the desired object is made through voxel reconstruction. After 3D model estimation non-object voxels are removed by space carving. At the end shape refinement and shape evolution steps are carried out to better fit the developed 3D model with the original object shape.

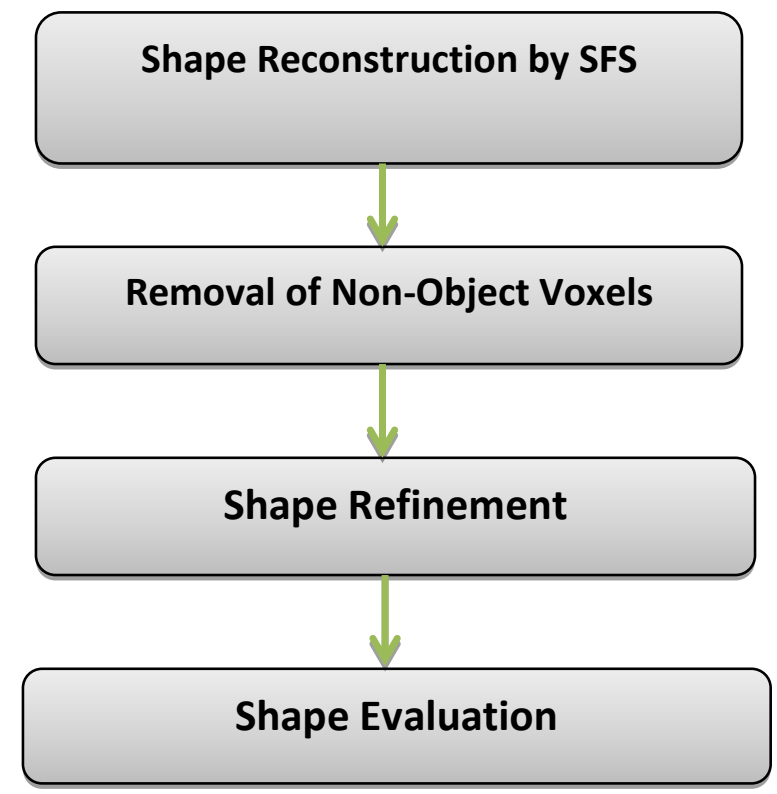

Fig. 4: Back End of SFS Pipeline

The major area of interest in this work is front end of SFS pipeline. Whereas the back end of SFS pipeline is regarded as 3D shape reconstruction step.

\section{RESULTS}

In this section results to the experiments to image sets have been discussed. First 3D model is achieved from image set of good quality and of high resolution. Later on 3D model was obtained from blur and degraded image set respectively. At the end $3 \mathrm{D}$ model is reconstructed from the image set obtained after the application of proposed model for blurriness and noise removal.

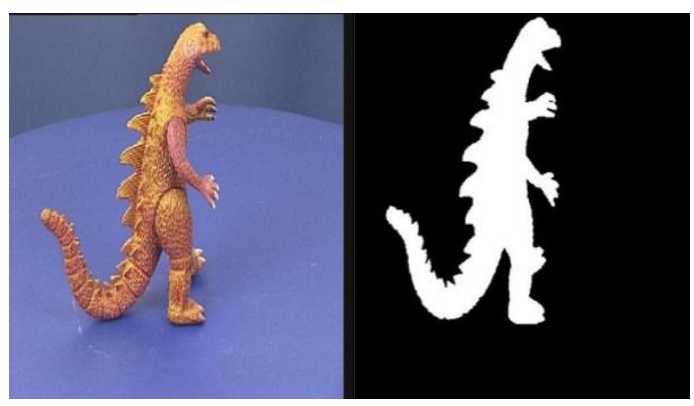


(a)
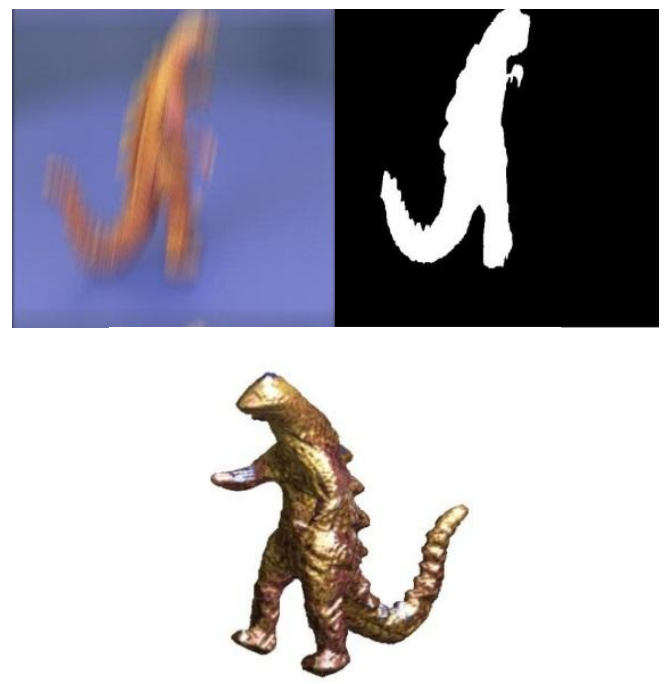

(b)
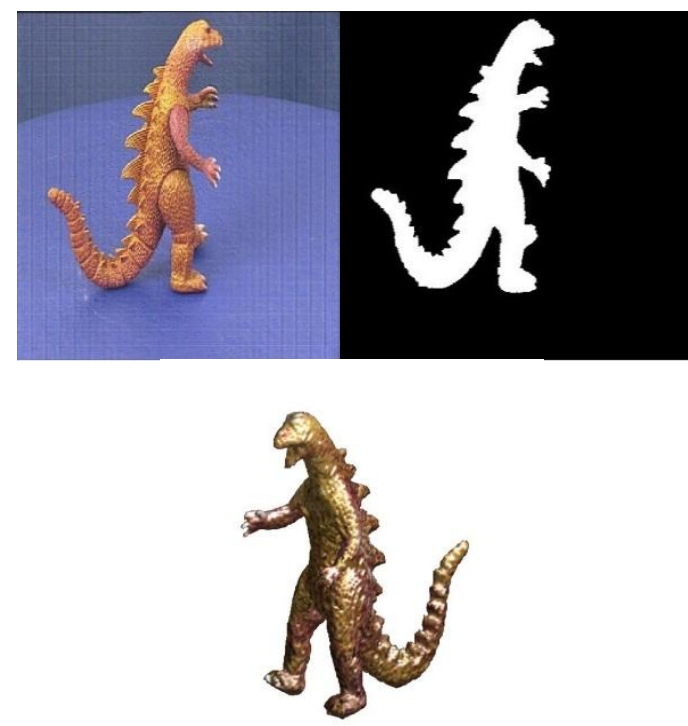

(c)

Fig. 5: Comparison of 3D shape reconstructed quality before, with and after blurriness removal

In Figure 5, Silhouette extracted in (a) from original pipeline without any degradation introduced in the image. The silhouette is perfect and hence the 3-D model generated at the end is well resembled to original object shape. In (b), blurred image, blurred kernel $(\mathrm{k})$ of 45 and orientation $(\theta)$ Of 25 is introduced, silhouette from the blurred image is extracted and 3-D model from the extracted silhouettes is shown. Here the extracted silhouette is very ambiguous, unable to represent the desired object shape, which shows in the presence of blurriness an object exact shape extraction is very hard task. At the end of the pipeline 3-D object obtained have some missing features. Both of the hands and lower jaw of dinosaur model are not reconstructed .In (c) image is obtained after removing blurriness using blind de-convolution. Here vertical lines are introduced after de-convolution but the shape of the object is clear and easily visualized. Hence the silhouette extracted from such image has close resemblance with the silhouette extracted from original degradation free image.

In this section results of noise removal module are discussed. Histogram of original image and image corrupted with noise of different densities has been analyzed and performance of proposed method for noise extermination is measured.

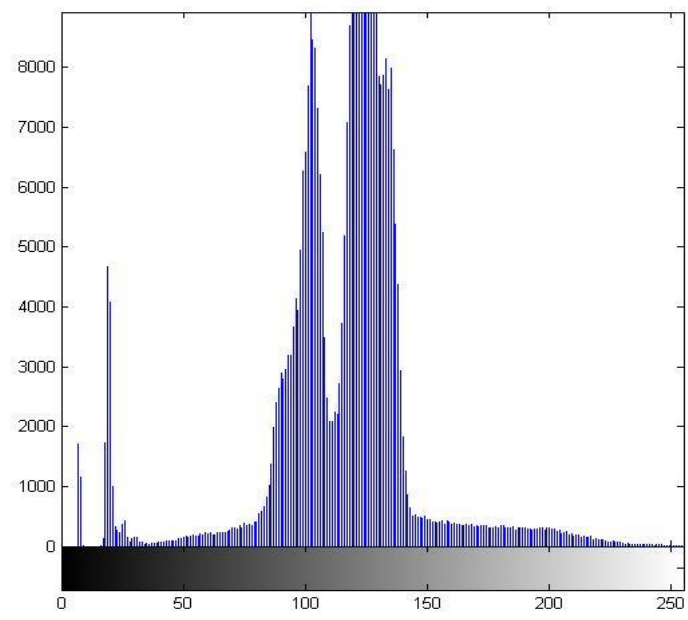

Fig. 6: Hitogram of Orignal Image

Figure 6, shows the histogram of original image. Image set used in this work has is rich in two main component colors. I.e. blue for the back ground and red more than blue for the foreground object. From the histogram graph in Figure 6, it is visible that the given image has one dominant component color i.e. red for the foreground object. And the background color i.e. blue is evenly distributed.

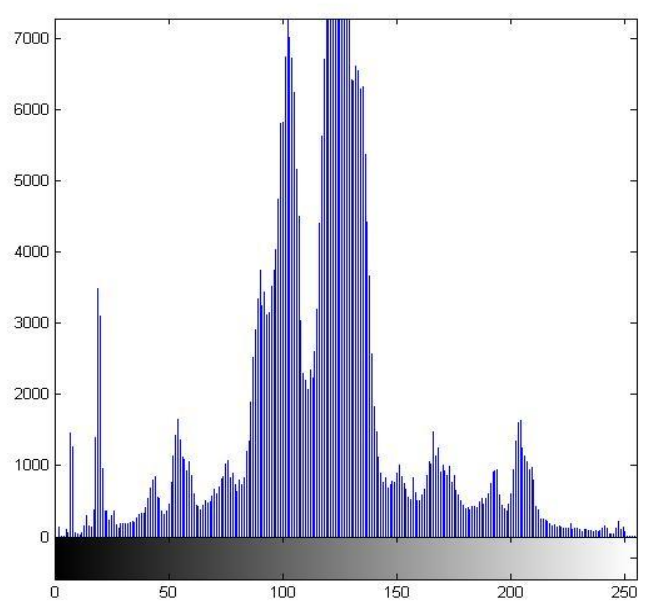

Fig. 7: Histogram of the Image corrupted with impulsive noise of density 0.1

As the density of impulsive noise is increased its effect in histogram graph is visible. There is variation in uniformly distributed histogram region as well foreground object region. Unwanted spikes in the uniformly distributed color region are visible in the histogram graph. In Figure 7, noise density is of 0.1 and as the density is further increased there is great 
deviation in original image histogram and the histogram of the noise image.

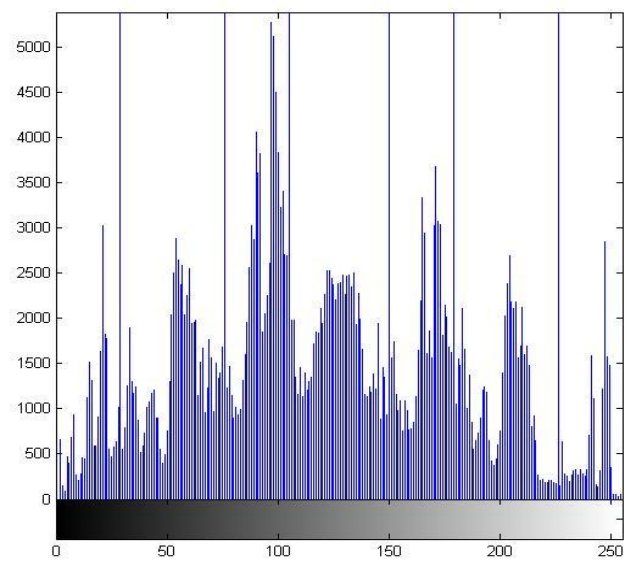

Fig. 8: Histogram of the Image corrupted with impulsive noise of density 0.3

In Figure 8, histogram of noise image with noise density of 0.3 has been plotted. From the histogram graph of the image, it is hard to understand image information. Histogram of the image deviates largely from that of original image. The deviation in image histogram indicates that noise has added extra unwanted information in the image. The drawback of this unwanted information added to the image will leads to imperfect segmentation process and as a result silhouette extracted from such imperfect segmented image will be inaccurate, because it is hard to identify that which is the region of interest. The addition of unwanted signals in the image has suppressed the foreground object region and has enhanced the background color area to almost that of foreground color area.

Figure 9 depicts the impact of silhouette extraction and 3-D model generation from image degraded with noise. Silhouette extracted in (b) from degraded image due to noise (a) is coarse. Noise density in the degraded images is 0.1 . The edges of extracted silhouette are rough and fail to give information about the exact boundaries of the observed object. While combining multiple views to estimate $3-\mathrm{D}$ volume is inaccurate. The pipeline fails to refine the object shape and color in accordance to the original object.

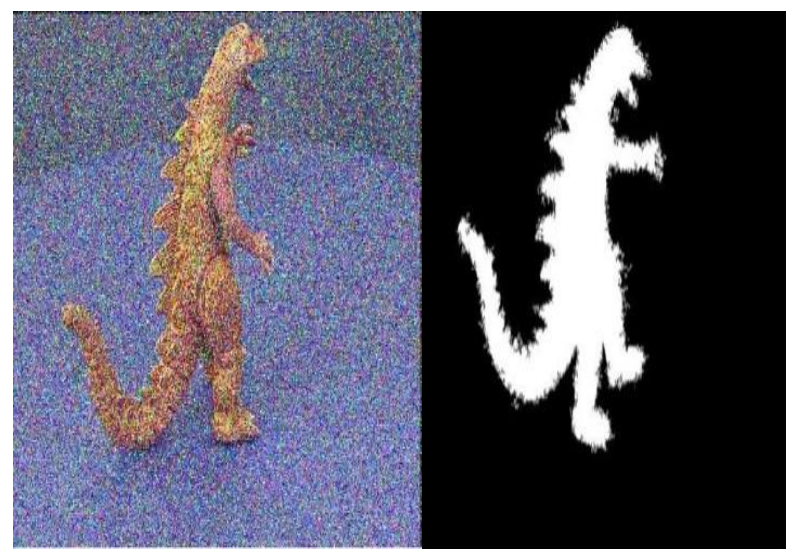

(a)

(b)

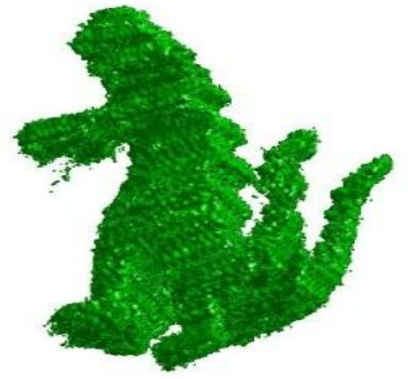

(c)

Fig. 9: Silhouette extraction and 3-D model reconstruction from noise degraded image

Figures 10, shows restored image through propose solution, extracted silhouette from the restored image and 3D model obtained from the restored image set. In restored image after the application of proposed median filter impulsive noise has been made minimal. Although noise is not fully exterminated, it can be seen in the background but the object in the image can easily be visualized. The segmentation step and silhouette extraction can be carried out easily without loss of useful information about the shape the object in the image. Hence the silhouette extracted is good representation of object shape. 3-D object obtained is well resembled to original object's shape.

\section{CONCLUSION}

So far in this work imaging in an uncontrolled environment is considered, which involves many degraded factors like blurriness, additive noise which badly affects the image resolution, illumination and precious information like shape description of an object in the images has also been lost due to induction of such degradations in the image set used for 3D modeling. Such images are unsuitable for silhouette extraction and 3D model reconstruction by Shapes from Silhouette technique.

Some preprocessing steps are introduced to tackle with such kind of degradations in an image set used for 3D modeling, due to which the processed images are made of such level that can be visually acceptable, free of degradation and are considered suitable for silhouette extraction and 3D model reconstruction.

From the results it is concluded that the proposed system worked well for enhancement of degraded images provided as input. The proposed system is effective against high density of noise and also for great amount blurriness in the image set used for 3D modeling. From this enhancement silhouette extraction from blurred, infected from additive noise which badly affects an image property become possible. A degraded image data of which reacquisition is not possible, by proposed system design can be made of that level which can be used to estimate well resembled 3-D model to that of real object shape.

As a future work, improvements to the proposed system design can be made, as this work can be considered at initial stage. This work can be combined with Shape from silhouette with un-calibrated camera. In uncontrolled environment, 
Photography through un-calibrated camera is carried out. This will be a positive addition to real time system development.
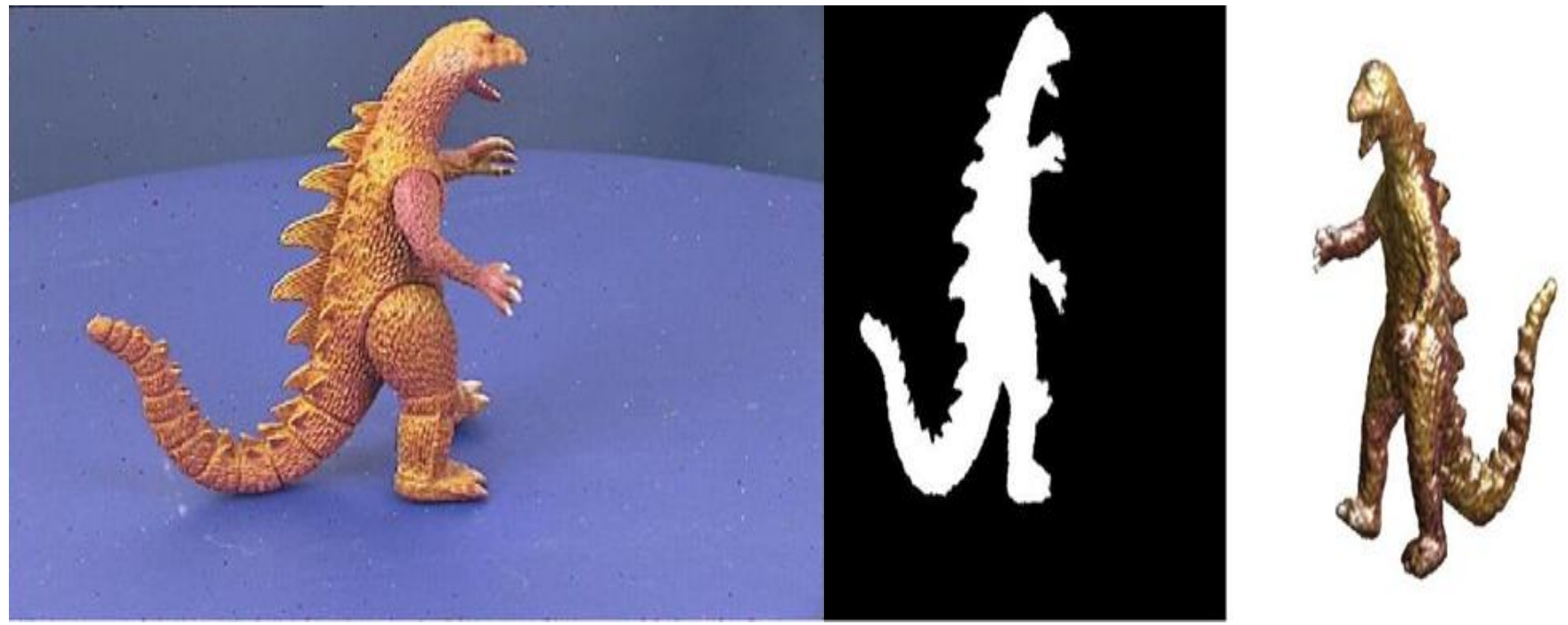

Fig. 10: Image restored after application of 2-D median filter to each color channel separately. Silhouette and 3-D model reconstructed from the restored image

\section{REFERENCES}

[1] Hirano, D, Funayama, Y, \& Maekawa, T. 2009. 3D Shape Reconstruction from 2D Images. Computer-Aided Design \& Applications, 6(5).

[2] Laurentini, A. 1994. The visual hull concept for silhouette-based image understanding. IEEE Transactions on Pattern Analysis and Machine Intelligence, 16(2), 150-162.

[3] Baker, S., \& Kanade, T. 2005. Shape-from-silhouette across time part i: Theory and algorithms. International Journal of Computer Vision, 62(3), 221-247.

[4] Kutulakos, K. N., \& Seitz, S. M. 2000. A theory of shape by space carving. International Journal of Computer Vision, 38(3), 199-218.

[5] Punjabi, V. D., Kumar, S., \& Gupta, N. 2012. A survey on removal of impulse noise from images by reducing execution time. World Journal of Science and Technology, 2(3).

[6] Wang, G., Li, D., Pan, W., \& Zang, Z. 2010. Modified switching median filter for impulse noise removal. Signal Processing, 90(12), 3213-3218
[7] Patil, P. A., \& Wagh, R. B. 2012. Review of blind image restoration methods. World Journal of Science and Technology, 2(3).

[8] Campisi, P., \& Egiazarian, K. (Eds.). 2007. Blind image deconvolution: theory and applications. CRC press.

[9] Robotics Research Group University of Oxford available online on: http://www.robots.ox.ac.uk.

[10] Tordoff, B., Carving a Dinosaur. Available online on, http://www.mathworks.com/matlabcentral/fileexchange/ 26160-carving-a-dinosaur, MatlabCentral.

[11] Krahmer, F., Lin, Y., McAdoo, B., Ott, K., Wang, J., Widemann, D., \& Wohlberg, B. 2006. Blind image deconvolution: Motion blur estimation. IMA Preprints Series, 2133-5.

[12] Zhu, X., Šroubek, F., \& Milanfar, P. 2012. Deconvolving PSFs for a better motion deblurring using multiple images. In Computer Vision-ECCV 2012 pp. 636-647. Springer Berlin Heidelberg.

[13] Cho, T. S., Paris, S., Horn, B. K., \& Freeman, W. T. June 2011. Blur kernel estimation using the radon transform. In IEEE Conference on Computer Vision and Pattern Recognition (CVPR). 241-248. 